\title{
THE UNIT SUM NUMBER OF SOME PROJECTIVE MODULES
}

\author{
NAHID ASHRAFI \\ Department of Mathematics, Semnan University, Semnan, Iran \\ e-mail: <nashrafi@semnan.ac.ir>, <ashrafi49@yahoo.com>
}

(Received 12 November, 2006; revised 12 September, 2007; accepted 14 October, 2007)

\begin{abstract}
The unit sum number $\mathbf{u}(R)$ of a ring $R$ is the least $k$ such that every element is the sum of $k$ units; if there is no such $k$ then $\mathbf{u}(R)$ is $\omega$ or $\infty$ depending whether the units generate $R$ additively or not. If ${ }_{R} M$ is a left $R$-module, then the unit sum number of $M$ is defined to be the unit sum number of the endomorphism ring of $M$. Here we show that if $R$ is a ring such that $R / \mathrm{J}(R)$ is semisimple and $\mathbb{Z}_{2}$ is not a factor of $R / \mathrm{J}(R)$ and if $P$ is a projective $R$-module such that $J P \ll P$, then $\mathbf{u}(P)=2$. As a result we can see that if $P$ is a projective module over a perfect ring then $\mathbf{u}(P)=2$.
\end{abstract}

2000 Mathematics Subject Classification. 13C10, 16D40, 16D10, 16G10, 16W20.

1. Introduction. The focus of this paper is on finding the unit sum number of a certain class of modules. All rings in this paper will have identity elements, all modules are unitary and considered as left $R$-modules. We use the symbol $\mathrm{U}(R)$ to denote the group of units of $R$ and $\mathrm{J}(R)$ for the Jacobson radical of $R$. We write the ring of $n \times n$ matrices over $R$ as $\mathrm{M}_{n}(R)$ and $\operatorname{End}_{R}(M)$ for the ring of endomorphisms of the $R$-module $M$.

The unit sum number of rings and modules was first introduced by B. Goldsmith et al. [4]. Recall that an element $r \in R$ is said to be $k$-good if $r=u_{1}+\cdots+u_{k}$ with $u_{1}, \ldots, u_{k} \in \mathrm{U}(R)$, and the ring $R$ is said to be $k$-good if every element of $R$ is $k$-good.

\section{DEFINITION 1.}

(i) For a ring $R$ the unit sum number $\mathbf{u}(R)$ is given as

- $\min \{k \mid R$ is $k$-good $\}$ if $R$ is $k$-good for some $k \geq 1$;

- $\omega$ if $R$ is not $k$-good for any $k$, but every element of $R$ is $k$-good for some $k$, (i.e. when at least $\mathrm{U}(R)$ generates $R$ additively);

- $\infty$ otherwise (i.e. when $\mathrm{U}(R)$ does not generate $R$ additively).

(ii) For an $R$-module $M$, the unit sum number of $M$ is denoted by $\mathbf{u}(M)$ and defined to be the unit sum number of $\operatorname{End}_{R}(M)$.

There is a considerable body of literature on this topic. Some are without the terminology used above including [2], [3], [4], [5], [6] and [7]. In 1954 Zelinsky [7] showed that for a vector space $V$ over a division ring $D, \mathbf{u}(V)=2$ unless the dimension of $V$ is 1 and $D$ is the field of two elements. Recently C. Meehan proved this statement for any free $R$-module when $\mathbf{u}(R)=2$. In fact he proved [6, Theorem 2.7] that if $m$ is a positive integer and a free $R$-module of finite rank $m$ has unit sum number equal to 2 then a free $R$-module of any infinite rank also has unit sum number equal to 2 . Here, by using the results above, we shall prove similar statements for some classes of projective modules. 
Definition 2. Let $M$ be an $R$-module and $K$ a submodule of $M$. We say $K$ is superfluous (or small) in $M$, written $K \ll M$ in case, for every submodule $L \leq M$, we have

$$
K+L=M \text { implies } L=M .
$$

Definition 3. Let $M$ be a left $R$-module. Then the radical of $M$ is defined as

$$
\operatorname{Rad}(M)=\bigcap\{K \leq M \mid K \text { is maximal in } M\} .
$$

From [1, Proposition 17.10] we know that if $R$ is a ring with $J(R)=J$ and $P$ is a projective module, then $\operatorname{Rad}(P)=J P$. In fact, we shall prove that if $R$ is a ring such that $R / \mathrm{J}(R)$ is semisimple and does not have $\mathbb{Z}_{2}$ as a factor and if $P$ is a projective $R$-module such that $J P \ll P,(J P$ small in $P)$, then $\mathbf{u}(P)=2$. As a result we can see that any projective $R$-module $P$ over a perfect ring that does not have $\mathbb{Z}_{2}$ as a factor has unit sum number 2; also if $R / \mathrm{J}(R)$ is semisimple and does not have $\mathbb{Z}_{2}$ as a factor and $P$ is a finitely generated projective module, then again, $\mathbf{u}(P)=2$.

2. The unit sum number of some projective modules. Before discussing the main results we need some properties of the unit sum number of rings and modules.

LEMMA 4. Let $R$ be a ring and let $I$ be an ideal of $R$. Then $\mathbf{u}(R / I) \leq \mathbf{u}(R)$ with equality if $I$ is contained in the Jacobson radical of $R$.

Proof. See [2, F2].

Note that since $\mathbf{u}\left(\mathbb{Z}_{2}\right)=\omega$, if $\mathbb{Z}_{2}$ is a factor of $R$ then, by Lemma 4 , the unit sum number of $R$ cannot be finite and it is $\omega$ or $\infty$. Hence having $\mathbb{Z}_{2}$ as a factor is a strong tool in determining whether $\mathbf{u}(R)$ is finite or not.

LEMMA 5. If the ring $R$ is a finite product of the rings $R_{1}, \ldots, R_{n}$, then $\mathbf{u}(R) \geq$ $\max \left\{\mathbf{u}\left(R_{1}\right), \ldots, \mathbf{u}\left(R_{n}\right)\right\}$, with equality holding if the right hand side is either finite or $\infty$.

Proof. See [2, F5].

LeMma 6. Let $R$ be a ring and ${ }_{R} M=\prod_{i=1}^{n} M_{i}$ a finite direct product of $R$-modules. For all $i \neq j$, let $\operatorname{Hom}\left(M_{i}, M_{j}\right)=0$. Then

$$
\mathbf{u}(M) \geq \max \left\{\mathbf{u}\left(M_{1}\right), \ldots, \mathbf{u}\left(M_{n}\right)\right\},
$$

with equality holding if the right hand side is either finite or $\infty$.

Proof. As $\operatorname{Hom}\left(M_{i}, M_{j}\right)=0$, for $i \neq j, \operatorname{End}_{R}(M)=\prod_{i=1}^{n} \operatorname{End}_{R}\left(M_{i}\right)$. Now, clearly by the definition of the unit sum number of modules and Lemma 5 , the result follows.

Proposition 7. Let ${ }_{R} X$ be a simple module and $R \cong X^{n}$, for some $n \in \mathbb{N}$, and let $M$ be an $R$-module. Then $\mathbf{u}(M)=2$ unless $M \cong X$ as modules and $\operatorname{End}_{R}(X) \cong \mathbb{Z}_{2}$ as rings.

Proof. As $X$ is simple it is clear that $R \cong X^{n}$ is semisimple. Let $F$ be a free $R$-module. There is a set $B$ such that $F \cong R^{(B)}$ and, since $R \cong X^{n}, F \cong X^{(S)}$ for some set $S$. As any $R$-module is a homomorphic image of a free $R$-module, by [1, Proposition 9.4] there is 
a set $A$ such that $M \cong X^{(A)}$, for some set $A$. If $|A|$ is infinite, then we have

$$
X^{(A)} \cong X^{\left(A^{n}\right)} \cong\left(X^{n}\right)^{(A)} \cong R^{(A)} .
$$

Therefore it is clear that ${ }_{R} M$ is a free $R$-module. Now, since $X$ is simple, $\operatorname{End}_{R}(X)$ is a division ring which we denote by $D$. Hence, viewed as rings,

$$
R \cong \operatorname{End}_{R}(R) \cong \operatorname{End}_{R}\left(X^{n}\right) \cong \mathrm{M}_{n}(D)
$$

By [4, Proposition 1.2(c)] the unit sum number of $R$ is 2. Therefore, by [6, Theorem 2.7], $\mathbf{u}(M)=2$ as well. Now if $|A|$ is finite, then for some integer $t, M \cong X^{t}$ and so $\operatorname{End}_{R}(M) \cong \operatorname{End}_{R}\left(X^{t}\right) \cong \mathrm{M}_{t}(D)$. Hence $\mathbf{u}(M)=2$.

Proposition 8. Let $D_{i}$ be a division ring, for every $i=1, \ldots, k$, and let $R=$ $\mathrm{M}_{n_{1}}\left(D_{1}\right) \times \mathrm{M}_{n_{2}}\left(D_{2}\right) \times \cdots \times \mathrm{M}_{n_{k}}\left(D_{k}\right)$. If $M$ is an $R$-module and $\mathbb{Z}_{2}$ is not a factor of $R$, then $\mathbf{u}(M)=2$.

Proof. By the theory of categories it is well known that if we denote the category of all $R_{1}$-modules by $R_{1} \mathcal{M}$ and the category of all $R_{2}$-modules by $R_{2} \mathcal{M}$ then we have

$$
R_{1} \mathcal{M} \times_{R_{2}} \mathcal{M} \cong_{R_{1} \times R_{2}} \mathcal{M}
$$

Now, since $M$ is an $R$-module, there are submodules $M_{i}$ which are $\mathrm{M}_{n_{i}}\left(D_{i}\right)$-modules, such that $M \cong M_{1} \times M_{2} \times \cdots \times M_{n}$ and $\operatorname{Hom}\left(M_{i}, M_{j}\right)=0$ for $i \neq j$. Now if for each $i$ we set $R_{i}=\mathrm{M}_{n_{i}}\left(D_{i}\right)$, then there are simple $R_{i}$-modules $X_{i}$ such that $R_{i} \cong X_{i}^{n_{i}}$ as $R_{i}$-modules. By Proposition $7, \mathbf{u}\left(M_{i}\right)=2$ for each $i$. Therefore $\mathbf{u}(M)=2$, by Lemma 5 .

All that remains is to prove the main theorem.

THEOREM 9. Let $R$ be a ring such that $R / \mathrm{J}(R)$ is semisimple and $\mathbb{Z}_{2}$ is not a factor of $R / \mathrm{J}(R)$. If $P$ is a projective $R$-module with $J P \ll P$, then $\mathbf{u}(P)=2$.

Proof. We write $J=\mathrm{J}(R)$ for the Jacobson radical of $R$. From [1, Corollary 17.12] we know that

$$
\operatorname{End}_{R}(P) / \mathrm{J}\left(\operatorname{End}_{R}(P)\right) \cong \operatorname{End}_{R}(P / J P) \cong \operatorname{End}_{R / J}(P / J P)
$$

As $R / J$ is semisimple, by Wedderburn's Second Theorem we know that $R / J$ is a finite direct product of simple Artinian rings. In fact there are division rings $D_{1}, \ldots, D_{k}$ such that $R / J \cong \prod_{i=1}^{k} \mathrm{M}_{n_{i}}\left(D_{i}\right)$. By Proposition 8 it is clear that $\mathbf{u}\left(\operatorname{End}_{R / J}(P / J P)\right)=2$. Hence $\mathbf{u}\left(\operatorname{End}_{R}(P) / \mathrm{J}\left(\operatorname{End}_{R}(P)\right)\right)=2$ and so, by Lemma $4, \mathbf{u}\left(\operatorname{End}_{R}(P)\right)=2$. This gives $\mathbf{u}(P)=2$.

In the following results we can see some classes of projective modules that satisfy the Main Theorem's conditions.

COROLlary 10. Let $R$ be a perfect ring and assume that $\mathbb{Z}_{2}$ is not a factor of $R$. If $P$ is a projective $R$-module, then $\mathbf{u}(P)=2$.

Proof. Since $R$ is a perfect ring, by [1, Theorem 28.4] we can conclude that $R / \mathrm{J}(R)$ is semisimple, where $\mathrm{J}(R)$ is left $T$-nilpotent, which implies that $J P \ll P$. Now, since $\mathbb{Z}_{2}$ is not a factor of $R$, it is not a factor of $R / J(R)$, so that the result clearly follows from Theorem 9 . 
COROLLARY 11. Let $R$ be a ring such that $R / \mathrm{J}(R)$ is semisimple and $\mathbb{Z}_{2}$ is not a factor of $R$. If $P$ is a finitely generated projective $R$-module, then $\mathbf{u}(P)=2$.

Proof. In this case, since $P$ is a finitely generated projective module, $J P \ll P$ and therefore the result follows from Theorem 9.

COROLlARY 12. Let $R$ be a semiprimary ring (i.e. $R / \mathrm{J}(R)$ is semisimple and $\mathrm{J}(R)$ is nilpotent). If $P$ is a projective $R$-module and $\mathbb{Z}_{2}$ is not a factor of $R$, then $\mathbf{u}(P)=2$.

Proof. Since $R$ is a semiprimary ring, we can see that $\operatorname{rad}(P)=J P \ll P$. Hence the result again follows from Theorem 9 .

\section{REFERENCES}

1. Frank W. Anderson and Kent R. Fuller, Rings and categories of modules, Graduate Texts in Mathematics, vol. 13 (Springer-Verlag, 1992).

2. Nahid Ashrafi and Peter Vámos, On the unit sum number of some rings, Quart. J. Math. Oxford Ser. (2) 56 (2005), 1-12.

3. Rüdiger Göbel and Ansgar Opdenhövel, Every endomorphism of a local Warfield module of finite torsion-free rank is the sum of two automorphisms, J. Algebra 233 (2000), no. 2, $758-771$.

4. B. Goldsmith, S. Pabst, and A. Scott, Unit sum numbers of rings and modules, Quart. J. Math. Oxford Ser. (2) 49 (1998), 331-344. 99-105.

5. Paul Hill, Endomorphism rings generated by units, Trans. Amer. Math. Soc. 141 (1969),

6. C. Meehan, Sums of automorphisms of free modules and completely decomposable groups, J. Algebra 299 (2006), 467-479.

7. Daniel Zelinsky, Every linear transformation is a sum of nonsingular ones, Proc. Amer. Math. Soc. 5 (1954), 627-630. 